\title{
Conservative Management of 12 Weeks Cervical Pregnancy following in vitro Fertilization
}

\author{
${ }^{1}$ PK Shah, ${ }^{2}$ Hemangi K Chaudhari, ${ }^{3}$ Neelima Mantri, ${ }^{4}$ Natasha D'Souza
}

\begin{abstract}
A 30-year-old, third gravid with two previous abortions, with in vitro fertilization conception with 11 weeks and 6 days of gestation, came with complaints of mild bleeding per vaginum. Ultrasonography revealed a live pregnancy in the cervical canal corresponding to 12 weeks of gestation. Single dose of intramuscular methotrexate $1 \mathrm{mg} / \mathrm{kg}$ was given. Three days postmedical management, transvaginal ultrasound-guided intracardiac instillation of potassium chloride $1.5 \mathrm{~mL}$ was carried out. Injection methotrexate, $4 \mathrm{~mL}$, was instilled in the amniotic cavity. On day 5 postprocedure, patient had a bout of severe vaginal bleeding for which emergency vaginal exploration was carried out. Products of conception were suctioned out and placenta was removed. Hemostatic cervical vascular sutures were taken on either side to occlude the descending branch of cervical artery. A Foley catheter $(18 \mathrm{~F})$ was inserted in the cervical canal and distended with $60 \mathrm{~mL}$ of normal saline, thereby compressing the placental bed from within the cervical canal. Hemostasis was achieved and maintained.
\end{abstract}

Keywords: Cervical ectopic pregnancy, Intracardiac potassium chloride, Methotrexate.

How to cite this article: Shah PK, Chaudhari HK, Mantri N, D'Souza N. Conservative Management of 12 Weeks Cervical Pregnancy following in vitro Fertilization. J South Asian Feder Obst Gynae 2017;9(2):185-189.

Source of support: Nil

Conflict of interest: None

Date of received: 2 January 2017

Date of acceptance: 17 March 2017

Date of publication: April 2017

\section{INTRODUCTION}

Cervical pregnancy represents a rare but life-threatening type of ectopic pregnancy. The incidence of cervical

${ }^{1}$ Professor, ${ }^{2}$ Associate Professor, ${ }^{3}$ Assistant Professor ${ }^{4}$ Speciality Medical Officer

${ }^{1-3}$ Department of Obstetrics and Gynecology, King Edward Memorial Hospital and Seth Gordhandas Sunderdas Medical College, Mumbai, Maharashtra, India

${ }^{4}$ Department of Obstetrics and Gynecology, Lokmanya Tilak Municipal General Hospital and Lokmanya Tilak Municipal Medical College, Mumbai, Maharashtra, India

Corresponding Author: Natasha D'Souza, Specialty Medical Officer, Obstetrics and Gynecology, Lokmanya Tilak Municipal General Hospital and Lokmanya Tilak Municipal Medical College Mumbai, Maharashtra, India, Phone: +8097807207, e-mail: natdsouza.87@gmail.com ectopic pregnancy is less than $1 \% .{ }^{1}$ Due to the advent of in vitro fertilization (IVF), there has been an increasing trend in cervical pregnancies in recent years. It is one condition in obstetrics where a single best modality of treatment has not yet been formulated.

\section{CASE REPORT}

A 30-year-old, third gravid with two previous abortions, IVF with 11 weeks and 6 days of gestation by last menstrual period, came to the emergency services with complaints of mild bleeding per vaginum and nonbilious vomiting since 1 day. She was diagnosed with hypothyroidism 3 years ago and was on treatment with Tab. levothyroxine for the same. Her husband had moderate oligozoospermia. The couple had miscarriage the previous two times at 6 to 8 weeks of gestation with spontaneous conception, for which emergency dilatation and curettage was performed. During this cycle, the couple had opted for donor oocytes and five embryos were transferred.

On examination, she was hemodynamically stable; there was no pallor, no abdominal guarding, or hemoperitoneum. Per speculum showed the cervix dilated, effaced anterior lip of cervix with $2 \times 2 \mathrm{~cm}$ bluish mass representing gestational mass inside the cervix. The mass did not bleed on touch. There was no evidence of fresh bleeding through the cervical os. Per vaginal examination was not done. Per rectal examination showed just bulky uterus with a $4 \times 4 \mathrm{~cm}$ lesion below the uterus. Bilateral fornices were uninvolved.

Necessary blood investigations were carried out and adequate blood was reserved. Her investigations revealed that hemoglobin was $11.4 \mathrm{gm} \%$, total leukocyte count was $10,600 / \mathrm{mm}^{3}$, platelet count was $3.1 \mathrm{lakh} / \mathrm{mm}^{3}$, serum creatinine $1.1 \mathrm{gm} / \mathrm{dL}$, and beta human chorionic gonadotropin (hCG) was $14,000 \mathrm{mIU} / \mathrm{mL}$. Coagulation profile was maintained. Ultrasonography revealed a live pregnancy in the cervical canal with placenta attached to the anterior cervical lip and crown-rump length corresponding to 12 weeks of gestation. Uterine fundal endometrium was collapsed with no gestational sac (Figs 1 and 2). Magnetic resonance imaging (MRI) confirmed the findings of ultrasonography (Fig. 3).

Conservative management approach was undertaken. Single dose of intramuscular methotrexate $1 \mathrm{mg} / \mathrm{kg}$ was 


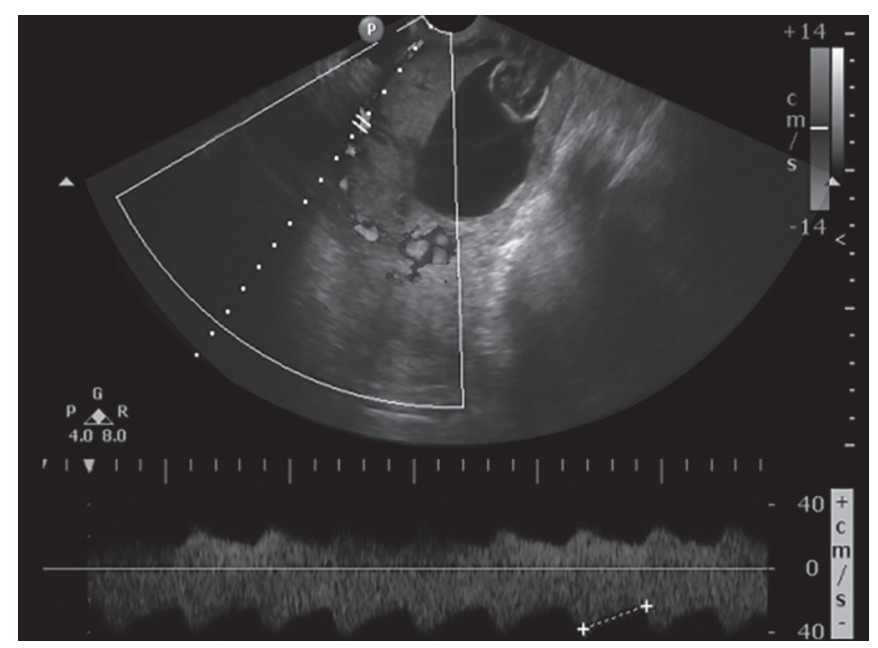

Fig. 1: Cervical ectopic gestational sac with fetus. ET: Endometrium

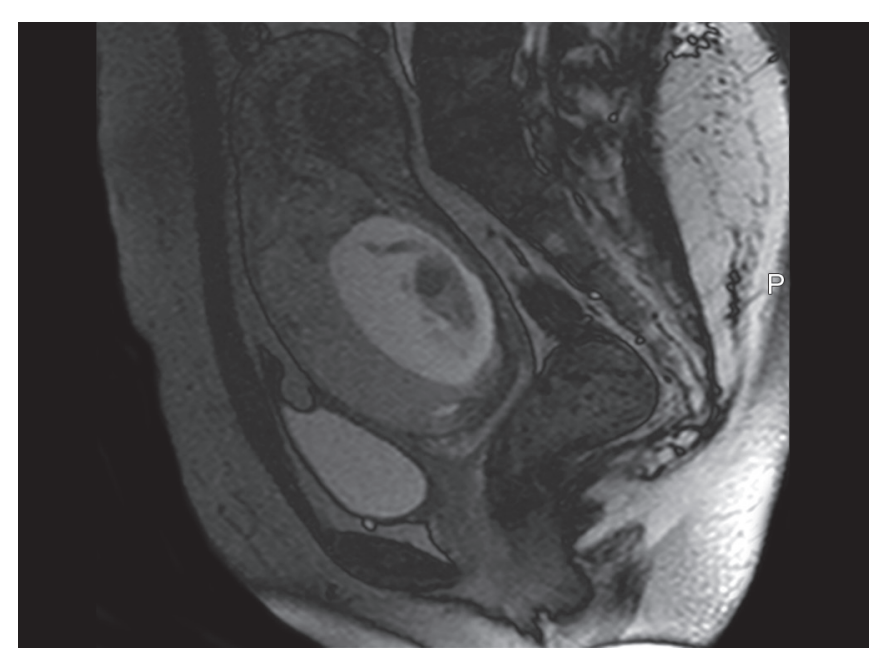

Fig. 3: Magnetic resonance imaging showing empty uterine cavity with gestational sac lying in the cervical canal

given and injection folinic acid $0.1 \mathrm{mg} / \mathrm{kg}$ was administered. Three days postmedical management, transvaginal ultrasound-guided intracardiac instillation of potassium chloride $1.5 \mathrm{~mL}$ was carried out, following which the fetal cardiac activity stopped and a crumpled fetal pole was observed. Amniotic fluid of 50 to $60 \mathrm{~mL}$ was aspirated and injection methotrexate, $4 \mathrm{~mL}$, was instilled in the amniotic cavity. The procedure was carried out with proper asepsis and did not result in active bleeding from the gestational sac except for minimal vaginal bleeding. Postprocedure ultrasonography findings were that the uterine artery peak systolic velocity was 46 , end diastolic velocity $=15$, and resistive index $=0.41$, sac measured $7 \times 3 \times 3.5 \mathrm{~cm}$, placenta measured $3.1 \mathrm{~cm}$, and fetal cardiac activity was absent with a crumpled fetal pole. There was no evidence of free fluid in the abdomen and the internal cervical os was closed. The findings are shown in Figures 4 to 6 .

Postprocedure, patient was keenly observed for changes in vital parameters and vaginal bleeding. Serum beta hCG levels and transvaginal ultrasound with color

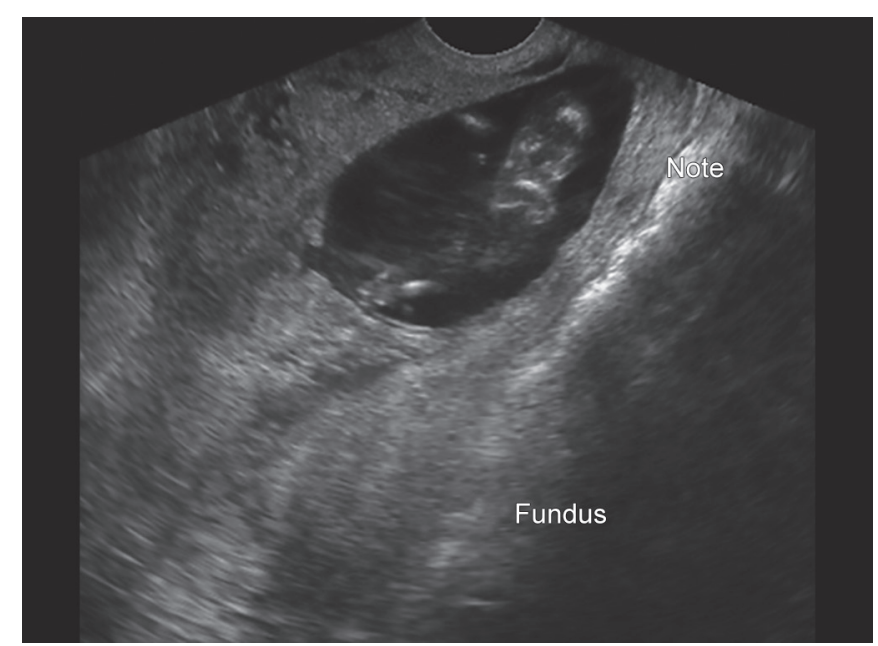

Fig. 2: Embryo in the cervical canal with normal fundus

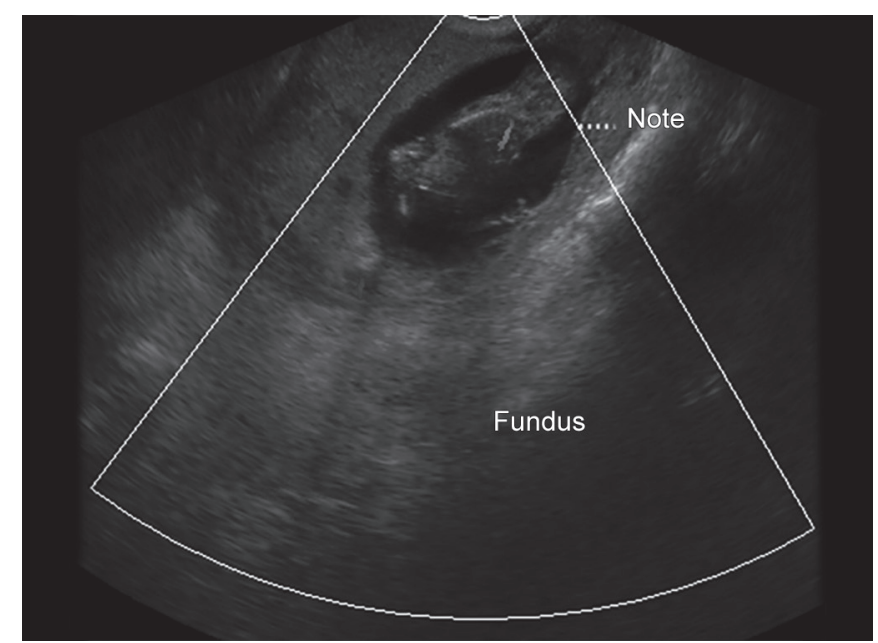

Fig. 4: Color Doppler showing cardiac activity

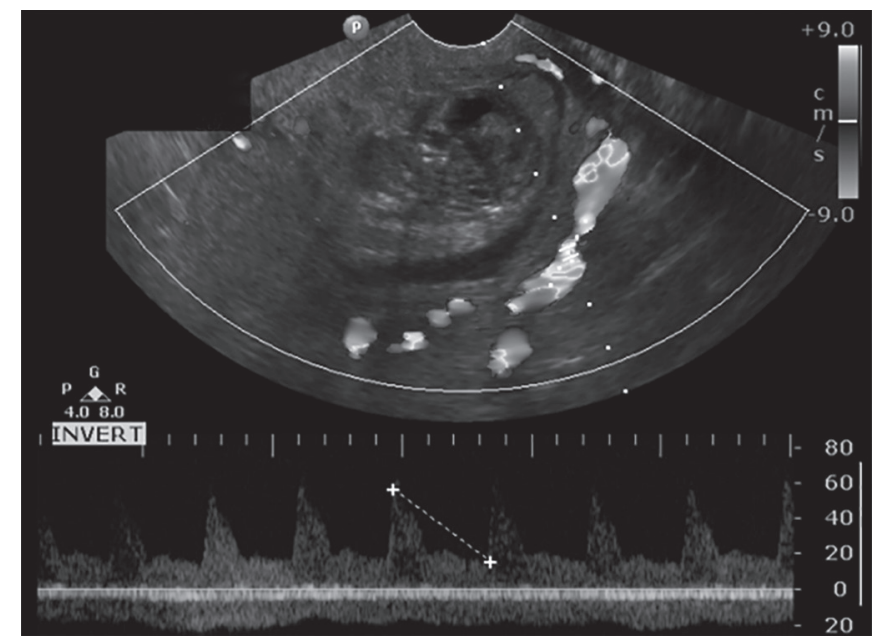

Fig. 5: Absent cardiac activity on color Doppler

Doppler were used to monitor therapy. Spontaneous abortion was awaited.

On day 5 postprocedure, patient had a bout of severe vaginal bleeding for which she was transfused blood and 


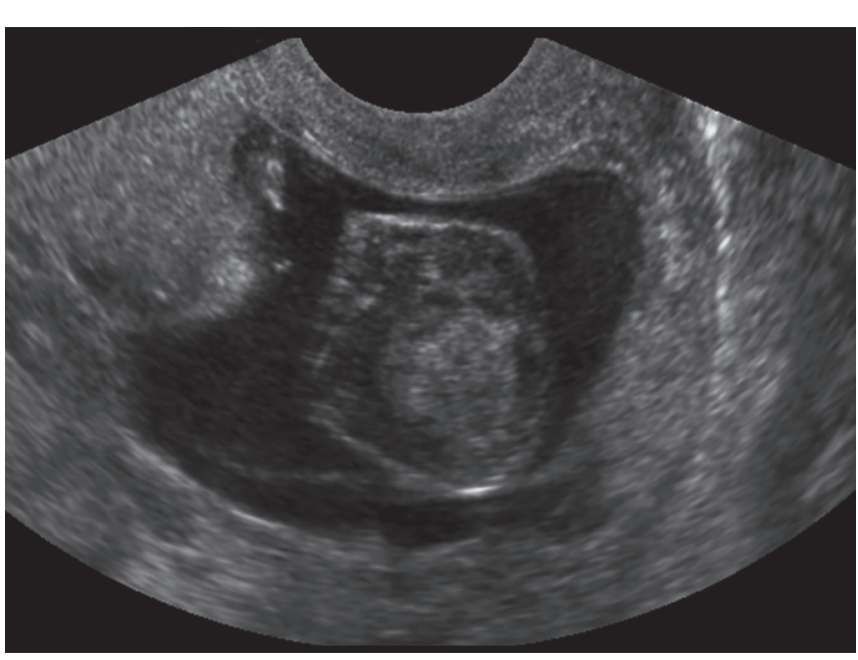

Fig. 6: Crumpled amniotic sac with reduced liquor

emergency vaginal exploration was carried out. Vaginal examination under anesthesia showed a mildly bulky uterus, firm in consistency, $5 \times 5 \mathrm{~cm}$ hour glass-shaped cervix with evidence of active bleeding from within the cervix with the placenta attached to the anterior lip of the cervix and the presence of fetal parts. Products of conception were suctioned out and placenta was removed. Hemostatic cervical vascular sutures were taken on either side to occlude the descending branch of cervical artery. Injection carboprost $250 \mu \mathrm{gm}$ was administered intramuscularly and Tab. misoprost $1000 \mu \mathrm{gm}$ was administered per-rectally. As hemostasis could not be achieved, it was decided to bring about internal and external cervical tamponade. A Foley catheter $(18 \mathrm{~F})$ was inserted in the cervical canal and distended with $60 \mathrm{~mL}$ of normal saline, thereby compressing the placental bed from within the cervical canal. This was followed by tight packing of the vagina with vaginal pack so as to bring about the external tamponade of the cervix. Hemostasis was achieved and maintained. Patient had approximate blood loss of $2.5 \mathrm{~L}$, which was promptly replenished with 6 units of packed cell blood transfusion intraoperatively and another 2 units in the postoperative period. Patient was monitored strictly and adequate antibiotic coverage was given.

After 48 hours of the procedure, the vaginal pack was removed. There was no evidence of bleeding. Five days after the procedure of cervical tamponade, the intracervical balloon was deflated in the operation theater to observe for any bleeding. As there was no active, fresh bleeding from the cervical canal, the Foley balloon was removed. Patient had minimal, foul-smelling vaginal discharge but the vaginal swab did not grow any organisms. Antibiotic coverage was continued. Patient did not have any fever in the postprocedure period. Her anemia was corrected and beta hCG was in the falling trend. Patient was discharged in stable condition on day 26 of admission, at which time beta hCG was $253 \mathrm{mIU} / \mathrm{mL}$.
Patient was followed up till the next menstrual cycle and no abnormalities were observed. Beta hCG were performed weekly till the normal range was obtained.

\section{DISCUSSION}

Cervical pregnancy is a rare form of ectopic pregnancy, which occurs in 1 in 1,000 to 1 in 18,000 pregnancies. The predisposing factors are instrumentation of the cervical canal, anatomic uterine anomalies, intrauterine device, and IVF. Literature also mentions exposure to diethylstilbestrol in utero as one of the causes, though the occurrence of this exposure is rare now. A rapid transport of the fertilized ovum into the cervix, before implantation in the endometrium due to an unreceptive endometrium has been postulated as one of the theories in the causation of cervical implantation.

Cervical pregnancy presents with a period of amenorrhea followed by painless vaginal bleeding.

If not diagnosed, it is an obstetrician's nightmare. In the past, the diagnosis of cervical pregnancy was made primarily after hysterectomy for uncontrolled bleeding. But more recently, the use of ultrasonography has facilitated early diagnosis, making provision for conservative management approach. The application of Doppler in differentiating cervical ectopic gestation from incomplete abortion is worth noting. Cervical gestation will show peritrophoblastic blood flow, whereas cervical abortion will not have it as it is separated from its vascular supply. In case of cervical abortion, the gestational sac is often described as crenated and with a dilated internal os on ultrasonography, whereas in cases of cervical ectopic, the gestational sac will be round or oval and may show a yolk sac or fetus with a closed internal os. The endometrial cavity will show some evidence of blood or products of conception in cases of cervical abortion, while cervical ectopic will show signs of decidual reaction. When cervical ectopic gestation is diagnosed early, ultrasound-guided termination or other conservative procedures allow preservation of the uterus, thus maintaining potential fertility.

Rubin, ${ }^{2}$ in 1911, formulated a criteria for the diagnosis of cervical ectopic gestation as follows:

- Cervical glands must be opposite to the site of placenta attachment.

- Placental attachment to the cervix must be situated below the entrance of the uterine vessels or below the peritoneal reflection of the anterior and posterior surfaces of the uterus.

- Fetal elements must be absent from the corpus uteri. The criteria to diagnose cervical ectopic gestation on ultrasonography were given by Hofmann et $\mathrm{al}^{3}{ }^{3}$ which is an hour glass shape of the uterus with cervical ballooning, empty uterine cavity, presence of gestational sac within 
the cervical canal and closed internal os, partially open external os with absence of a sliding sac sign.

The modalities of conservative medical management available are systemic methotrexate with single- or multiple-dose regime, intraamniotic methotrexate, intracardiac potassium chloride, or a combination of any of these. The major drawback of intraamniotic injection technique is the skill and radiological expertise. The amniotic sac must be kept intact to avoid substantial bleeding. Surgical procedures available are dilatation and curettage, intracervical balloon tamponade, cervical stay sutures, internal iliac artery ligation, uterine artery embolization (UAE), or, the last resort, hysterectomy. Lower hCG levels, gestational age less than 12 weeks, and absence of cardiac activity have a better prognosis with conservative management. Thus, these parameters need to be considered to guide the treatment regime.

Surampudi ${ }^{4}$ reported a case of cervical ectopic pregnancy of 6 weeks gestation managed successfully with systemic methotrexate, two doses at an interval of 1 week, due to inadequate fall in beta hCG levels. Patient achieved normal levels of the hormone in 5 weeks following the second dose with complete resolution of ultrasonography. In contrast to the above case scenario, our case did not respond to the medical management. The causes of this failure could be postulated as advanced gestation, presence of cardiac activity, and high levels of beta hCG.

In 2014, Sijanović et $\mathrm{al}^{5}$ reported a case of cervical ectopic pregnancy, which was unsuccessfully managed by single dose of local, ultrasound-guided intraamniotic methotrexate. Though the follow-up after methotrexate therapy showed decreasing trend in beta hCG levels and collapsed gestational sac on ultrasonography, on the 30th day of methotrexate therapy, patient had persistent vaginal bleeding and on 37th day patient had moderate vaginal bleeding. While conducting vaginal examination, patient had torrential bleeding, requiring temporary cervical tamponade and hysteroscopic resection of conceptional products from the posterior lip of the cervix. The researchers concluded that methotrexate in combination with other minimally invasive surgical procedures can lead to a satisfactory outcome, thereby preserving fertility.

A retrospective observational study was performed by Zakaria et $\mathrm{al}^{6}$ to evaluate the role of UAE in the management of cervical pregnancy, wherein patients were treated with methotrexate alone $(n=5)$, UAE in conjunction with methotrexate $(\mathrm{n}=6)$, and a third group with intraamniotic potassium chloride and UAE $(n=4)$. They observed that patients with statistically significant higher beta hCG values had received UAE. Out of 10 patients who underwent UAE, 2 patients had cervical ectopic pregnancies with presence of cardiac activity at the time of presentation. It was concluded that UAE with methotrexate is an option for minimally invasive intervention in the treatment of cervical ectopic pregnancy. Another study by Hirakawa et $\mathrm{al}^{7}$ to evaluate the technical and clinical outcome of UAE in the management of cervical ectopic pregnancy demonstrated that all eight patients could be managed by UAE in conjunction with methotrexate. No patient required hysterectomy. At follow-up, three patients had subsequent successful natural pregnancies.

Hysterectomy was considered as the mainstay therapy in the old literature of the management of cervical ectopic pregnancy, but the author proposes that hysterectomy as a management option for cervical ectopic gestation should be reserved for cases not responding adequately to all other conservative, noninvasive modalities of management as it sacrifices the patient's future obstetric career. Many studies have shown that cervical ectopic pregnancy can be managed without hysterectomy. Verma and Goharkhay ${ }^{8}$ reported a case series of 24 cases of cervical ectopic pregnancy, all of which were managed satisfactorily without requiring hysterectomy. This approach also led to successful salvage of the live intrauterine pregnancy in two cases of heterotropic gestation. The easy availability of ultrasonography has helped drastically in the identification of cases at an earlier gestation and at a stage where complications have not yet occurred, thus enabling use of medical and minimally invasive treatment options. The advancement in the diagnosis has narrowed down the need of hysterectomy for cervical ectopic gestation by allowing the preparation and implementation of a conservative approach in a more controlled manner. The indication where hysterectomy is necessary is cervical ectopic reaching second or third trimester of gestation, which itself is rare in the current era of modern obstetrics.

Although conservative management approach appears to be superior in the majority of patients, hysterectomy is a relatively less expensive option as compared with other options of the spectrum of conservative approach; thus, hysterectomy may be considered in women nondesirous of future fertility.

\section{CONCLUSION}

Though a rare condition, advent of assisted reproductive techniques has resulted in an increased occurrence of cervical ectopic pregnancy. A surgeon should have a differentiation of cervical ectopic pregnancy in cases of incomplete abortion to prevent a surgical catastrophe and to salvage the reproductive career of the patient. Precise diagnosis will reduce the maternal morbidity, including the need for hysterectomy and blood transfusion. Every intervention has proved to be a cornerstone in the successful management of cervical ectopic, as depicted in our case, resulting in restoring fertility and reducing maternal morbidity. Lastly, although a spectrum of management 
options are available, consensus is yet to be arrived on a definite management protocol. The basic management revolves around the attainment of hemostasis following evacuation of cervical ectopic gestation by UAE or local tamponade of the cervical bed or in intractable hemorrhage, hysterectomy may have to be performed.

\section{REFERENCES}

1. Cepni I, Ocal P, Erkan S, Erzik B. Conservative treatment of cervical ectopic pregnancy with transvaginal ultrasoundguided aspiration and single-dose methotrexate. Fertil Steril 2004 Apr;81(4):1130-1132.

2. Rubin IC. Cervical pregnancy. Surg Gynecol Obstet 1911;13: 625-633.

3. Hofmann HM, Urdl W, Höfler H, Hönigl W, Tamussino K. Cervical pregnancy: case reports and current concepts in diagnosis and treatment. Arch Gynecol Obstet 1987:241(1):63-69.
4. Surampudi K. A case of cervical ectopic pregnancy: successful therapy with methotrexate. J Obstet Gynaecol India 2012 Dec;62 (Suppl 1):1-3.

5. Sijanović S, Vidosavljević D, Topolovec Z, Milostić-Srb A, Mrčela M. Management of cervical ectopic pregnancy after unsuccessful methotrexate treatment. Iran J Reprod Med 2014 Apr;12(4):285-288.

6. Zakaria MA, Abdallah ME, Shavell VI, Berman JM, Diamond MP, Kmak DC. Conservative management of cervical ectopic pregnancy: utility of uterine artery embolization. Fertil Steril 2011 Mar;95(3):872-876.

7. Hirakawa M, Tajima T, Yoshimitsu K, Irie H, Ishigami K, Yahata H, Wake N, Honda H. Uterine artery embolization along with the administration of methotrexate for cervical ectopic pregnancy: technical and clinical outcomes. AJR Am J Roentgenol 2009 Jun;192(6):1601-1607.

8. Verma U, Goharkhay N. Conservative management of cervical ectopic pregnancy. Fertil Steril 2009 Mar;91(3):671-674. 